\title{
Independent Functions of Slit-Robo Repulsion and Netrin- Frazzled Attraction Regulate Axon Crossing at the Midline in Drosophila
}

\author{
David S. Garbe and Greg J. Bashaw \\ ${ }^{1}$ Department of Neuroscience, University of Pennsylvania School of Medicine, Philadelphia, Pennsylvania 19104
}

\begin{abstract}
Slit and Netrin and their respective neuronal receptors play critical roles in patterning axonal connections in the developing nervous system by regulating the decision of whether or not to cross the midline. Studies of both invertebrate and vertebrate systems support the idea that Netrin, secreted by midline cells, signals through DCC (Deleted in Colorectal Carcinoma)/UNC40/Frazzled receptors to attract commissural axons toward and across the midline, whereas Slit signals through Robo family receptors to prevent commissural axons from recrossing the midline, as well as to prevent ipsilateral axons from ever crossing. Recent evidence from both Xenopus neuronal cell culture and Drosophila genetics have suggested that these signals may interact more directly in a hierarchical relationship, such that one response extinguishes the other. Here we present loss- and gain-of-function genetic evidence showing that the influence of Slit and Netrin on midline axon crossing is dictated by both independent and interdependent signaling functions of the Robo and Frazzled (Fra) receptors. Our results are not consistent with the proposal based on genetic analysis in Drosophila that the sole function of Slit and Robo during midline guidance is to repress Netrin attraction.
\end{abstract}

Key words: axon guidance; midline; repulsion; attraction; Slit; Robo; Netrin; DCC/Frazzled

\section{Introduction}

Slit and Netrin and their receptors, Robo/Sax3 and Deleted in Colorectal Cancer (DCC)/Frazzled/UNC40, play critical and conserved roles in patterning axonal connections during development of the Caenorhabditis elegans, Drosophila, and vertebrate nervous systems (Dickson, 2001; Yu and Bargmann, 2001; Garbe and Bashaw, 2004). Studies of these systems support the idea that Netrin-DCC signaling predominantly mediates axon attraction, whereas Slit-Robo signaling mediates repulsion. In the Drosophila ventral nerve cord and vertebrate spinal cord, Slit and Netrin are secreted by midline cells (Serafini et al., 1994; Mitchell et al., 1996). In these contexts, Netrin mediates axon attraction across the midline, whereas Slit prevents inappropriate crossing (Serafini et al., 1996; Kidd et al., 1998a; Brose et al., 1999; Kidd et al., 1999; Long et al., 2004).

Two general proposals for how these signals are coordinated during midline guidance have emerged. Classically, it has been proposed that guidance decisions at intermediate targets, such as the midline, are shaped by the balance of attractive and repulsive cues to which the growth cone is exposed (Tessier-Lavigne and

Received Dec. 15, 2006; revised Feb. 27, 2007; accepted Feb. 27, 2007.

This work was supported by a Burroughs Wellcome Career Award and National Institutes of Health Grant NS046333 to G.J.B. We are grateful to members of the Bashaw laboratory for constructive comments, technical assistance, and support. We thank Corey Goodman, in whose laboratory the anti-Frazzled monoclonal antibody was generated.

Correspondence should be addressed to Greg J. Bashaw, Department of Neuroscience, University of Pennsylvania School of Medicine, 1113 BRB2/3, 421 Curie Boulevard, Philadelphia, PA 19104. E-mail: gbashaw@mail.med.upenn.edu.

DOI:10.1523/JNEUROSCI.0301-07.2007

Copyright $\odot 2007$ Society for Neuroscience $\quad$ 0270-6474/07/273584-09\$15.00/0
Goodman, 1996). An alternative, although not mutually exclusive, idea is that attraction and repulsion are sometimes more intimately linked to ensure robust responses and to prevent conflicting signals from confusing the growth cone. In this case, activation of one receptor directly inhibits the function of another. For example, in cultured Xenopus spinal neurons, Slit silences the attractive function of Netrin through induction of a direct receptor-receptor interaction between Robo and DCC (Stein and Tessier-Lavigne, 2001). These findings have raised questions about how much of Slit-Robo function is attributable to axon repulsion as opposed to the inhibition of attraction. Indeed, two recent reports in Drosophila have suggested that the major (or in one case the only) function of Slit and Robo during midline guidance is to inhibit responses to Netrin (Bhat, 2005; Hiramoto and Hiromi, 2006). For example, double mutants between slit and Netrin (the ligands) or robo and fra (the receptors) were reported to result in phenotypes that were indistinguishable from single Netrin and fra mutants, leading to the argument that the only function of Slit-Robo signaling is to inhibit Netrin-Fra attraction (Bhat, 2005).

Here, we have examined how Slit repulsion and Netrin attraction are integrated during midline guidance in an effort to resolve whether Slit-Robo signaling acts to repel axons at the midline or whether instead it is solely required to inhibit Netrin-mediated attraction. Analysis of multiple double-mutant combinations of the attractive and repulsive ligands and receptors directly contradicts the previous findings of Bhat (Bhat, 2005). Instead, we observe a combination of both phenotypes: some axons fail to cross the midline, whereas others abnormally cross. Reintroducing ei- 
ther Robo or Fra into the double mutants restores the phenotype to that observed in single mutants. In addition, we demonstrate that postcrossing axons recross the midline in robo mutants and that they do so independently of Netrin-fra function, suggesting that if silencing attraction is required to prevent axon recrossing in the Drosophila CNS, Netrin cannot be the sole arbiter of the attraction.

\section{Materials and Methods}

Genetics and molecular biology. The following stocks were used for this study: (1) $\mathrm{fra}^{3} / \mathrm{CyWg} \beta \mathrm{Gal}$, (2) $\mathrm{fra}^{4} / \mathrm{CyWg} \beta \mathrm{Gal}$, (3) $\mathrm{robo}^{\mathrm{Ga} 285} / \mathrm{CyWg} \beta \mathrm{Gal}$, (4) fra3, robo ${ }^{G a 285} / \mathrm{CyWg} \beta G a l$, (5) $\mathrm{fra}^{4}, \mathrm{robo}^{\mathrm{Ga285}} / \mathrm{CyWg} \mathrm{WGal}$, (6) Df NetrinA, B (NP5)/FM7 $\beta_{\text {actin }}$, (7) slit ${ }^{2} / C y W g \beta G a l$, (8) UAS-TauMycGFP/ CyTubulinGal80; eagleGal4, (9) fra ${ }^{4}$, UAS-TauMycGFP/CyTubulinGal80; eagleGal4, (10) robo ${ }^{\text {Ga285 }}$, UAS-TauMycGFP/CyTubulinGal80; eagleGal4, (11) robo ${ }^{G a 285}$, UAS-TauMycGFP/CyWgßGal, (12) robo ${ }^{\text {Ga285, }}$, apterousGal4/CyWg $\beta G a l$, (13) fra ${ }^{4}$, robo $^{\text {Ga285 }}$, apterousGal4/CyWg $\beta G a l$, (14) $\mathrm{fra}^{3}, \mathrm{robo}^{\mathrm{Ga} 285}, \mathrm{UAS}-\mathrm{TauMycGFP} / \mathrm{CyWg} \beta \mathrm{Gal}$, (15) fra ${ }^{4}, \mathrm{robo}^{\mathrm{Ga} 285}$ / CyWgßGal; elavGal4, (16) $\mathrm{fra}^{3}$, robo ${ }^{\mathrm{Ga} 285} / \mathrm{CyWg \beta Gal;}$ UAS-RoboMyc\#4, (17) $\mathrm{fra}^{3}$, robo ${ }^{\mathrm{Ga} 285} / \mathrm{CyWg} \beta$ Gal; UAS-FraMycII, (18) $\mathrm{fra}^{3}, \mathrm{robo}^{\mathrm{Z14} /}$ CyWgßGal, (19) $\mathrm{fra}^{4}$, robo ${ }^{Z 14} / \mathrm{CyWg} \beta$ Gal, (20) UAS-RoFra ${ }^{2 X}$ insert,$(21)$ NetA,B/FM7 $\beta_{\text {actin }}$. We took advantage of the $\beta$-galactosidase expression associated with the balancer chromosomes together with antibody or mRNA in situ analysis to unambiguously genotype mutant embryos.

To generate UASFramyc, an EcoRI site was included 5' of the fra coding sequence with the following primer: CAA ATA GAA TTC GCA ATC GGC GAT TGG CGG. A BamHI site was also included at the $3^{\prime}$ of the fra coding sequence to eliminate the STOP codon and clone fra in frame with a 6-myc tag followed by a STOP codon into a pUAST vector (sequence at the junction EFEC/SRIRAGS). UASFraMyc transgenic flies were generated according to standard procedures.

Antibody generation and immunohistochemistry. The following primary antibodies were used: (1) mouse (Ms) anti-1D4/FasII [Developmental Studies Hybridoma Bank (DSHB); 1:100], (2) Ms-monoclonal antibody (MAb) BP102 (1:100; DSHB), (3) rabbit (Rb)-anti-Myc (1:500; Sigma-Aldrich, St. Louis, MO), (4) Ms-anti-Slit (1:25; C555.6D; DSHB), (5) Ms-anti-Sex Lethal (1:1000; M18; DSHB), (6) Ms-anti- $\beta$ gal (40-1a; 1:250; DSHB), (7) Rb-anti-GFP (1:500; Invitrogen, Eugene, OR), (8) Ms-anti-Robo (1:50; DSHB), (9) Rb-anti-HRP (1:2000; MP Biomedicals, Solon, $\mathrm{OH})$. A mouse monoclonal antibody was generated against the Fra protein (amino acids 440-902) as described previously and used at 1:50. The following secondary antibodies were used: (1) Alexa Fluor 488 goat anti-Rb (1:500; Invitrogen), (2) cyanine 3 (Cy3) goat anti-Ms (1:1000; Jackson ImmunoResearch, West Grove, PA). Embryos were fixed and stained as described previously (Kidd et al., 1998a). Stacks of images were obtained using a Leica (Nussloch, Germany) DMIRE2 confocal and a $63 \times$ oil-immersion objective. A maximum projection of the stacks was generated with NIH Image/ImageJ software.

Fluorescence mRNA in situ. Embryo collection and in situ hybridization were performed as described previously with digoxigenin-labeled probes (Tear et al., 1996). The NetrinA and NetrinB in situ probes were PCR amplified and transcribed from full-length cDNAs cloned into pBluescript. Hybridized probes were detected with anti-digoxigeninHRP (Roche Diagnostics, Indianapolis, IN) and cyanine 5-labeled tyramide (see Fig. 2) or fluorescein-labeled tyramide (supplemental Fig. 2, available at www.jneurosci.org as supplemental material) (TSA Fluorescence System; PerkinElmer Life Sciences, Waltham, MA) was used as a substrate. After in situ hybridization, embryos were immunostained with anti-SlitC and anti-HRP as shown in Figure 2 or anti-Myc and anti-FasII as shown in supplemental Figure 2 (available at www.jneurosci.org as supplemental material). Stacks of images were obtained and processed as described above.

\section{Results}

To investigate the relationship between attraction and repulsion during midline axon guidance, we generated fly strains carrying various combinations of loss-of-function mutations in slit, robo, fra, and Netrin. In Drosophila, Netrin is encoded by two genes,
NetrinA and NetrinB; hereafter, we refer to the two genes as $\mathrm{Ne}$ trin for simplicity. Initially, we analyzed double-mutant embryos using BP102 as a pan-axonal marker or anti-FasII to recognize ipsilateral axons and compared their phenotypes to wild-type animals and single mutants (Fig. 1). In wild-type Drosophila embryos, immunostaining with the BP102 MAb reveals a ladder-like axon scaffold with longitudinal axons forming the rails of the ladder and commissural axons forming the rungs, whereas antiFasII staining reveals three parallel bundles of longitudinal axons on either side of the midline (Fig. 1 $A, E$ ). The attractive Netrin receptor Fra is expressed broadly and at high levels on many axons in the CNS, including both commissural and longitudinal portions of axons, whereas the Robo receptor is expressed broadly and at high levels along the longitudinal axon connectives but is kept at low levels on the commissural portions of axons (Fig. 1 I,M) (Kolodziej et al., 1996; Kidd et al., 1999).

Mutations in fra that eliminate Fra protein expression result in reduced or absent axon commissures as well as characteristic breaks in the longitudinal connectives; however, many axons still cross the midline normally in fra mutants, and the majority of the crossing defects are observed in the posterior commissure (Fig. $1 B, F, J)$ (see below) (Kolodziej et al., 1996; Forsthoefel et al., 2005). The longitudinal restricted expression pattern and levels of Robo are unaltered in fra mutants, indicating that loss of fra does not influence the repulsive pathway at the level of receptor expression or localization (Fig. 1N). Protein null mutations in robo lead to the opposite kind of phenotype, in which too many axons cross the midline. Axon commissures appear thicker than wild-type and are often fused together, whereas FasII-positive neurons are observed to wander back and forth across the midline (Fig. 1C,G) (Kidd et al., 1998a). In contrast to fra, in which mutant phenotypes show a high degree of segment-to-segment variability, essentially all segments in all embryos show defects in robo mutants (Fig. 1C,G) (Kidd et al., 1998a). We also tested whether Fra protein expression was in any way dependent on robo function and found no apparent differences in either levels or localization (Fig. $1 K$ ).

\section{Simultaneous reduction of attraction and repulsion results in guidance defects that combine aspects of both single-mutant phenotypes}

In light of the previously published data indicating that fra, robo double mutants are indistinguishable from fra single mutants (Bhat, 2005), we were surprised to find that embryos with mutations in fra and robo clearly exhibit defects that combine aspects of both mutant phenotypes; with some segments exhibiting the thickened and fused commissures characteristic of robo mutants and some segments showing thin or missing posterior commissures characteristic of fra mutants (Fig. $1 D, L, P$ ). This is true of multiple different allelic combinations of fra and robo (see Materials and Methods). In addition, clear defects in midline crossing of FasII-positive axons are observed in the double mutants, although these crossing defects do not appear to be as severe as robo single mutants (Fig. $1 H$ ). Importantly, immunostaining confirms the absence of both Fra and Robo proteins in these double mutants (Fig. $1 L, P$ ). Furthermore, out-crossing our fra, robo double mutants to either fra or robo single mutants resulted in phenotypes that were identical to the single-mutant fra and robo phenotypes described above (Fig. 1).

We next extended our analysis to an examination of Netrin; slit and fra, slit double mutants and found that both of these double mutants much more closely resemble the slit singlemutant phenotype in which all axons collapse onto and grow 
along the CNS midline (Fig. 2) (data not shown). In these experiments, two different strategies were used to eliminate Netrin function. First, we used an $\mathrm{X}$-chromosomal deletion that is known to completely remove both Netrin genes, as well as several additional genes (Mitchell et al., 1996). Second, we used specific NetAB double mutants generated by homologous recombination (Brankatschk and Dickson, 2006). In both types of Netrin; slit double mutants, we confirmed the absence of Netrin and Slit by fluorescence mRNA in situ and immunostaining, respectively (Fig. $2 \mathrm{H}, \mathrm{J}$ ) (data not shown). In contrast to the fra, robo double mutants, in Netrin, slit or fra, slit double mutants it was more difficult to discern aspects of the fra and Netrin phenotypes (Fig. 2D). This is likely attributable to the fact that when only robo1 is absent, robo 2 can provide some repulsive activity, whereas in slit mutants, the repulsion is completely lost (Kidd et al., 1999; Rajagopalan et al., 2000; Simpson et al., 2000). Consistent with this idea, fra, robo, robo 2 triple mutants showed phenotypes that were very similar to those seen in fra, slit double mutants (data not shown). Nevertheless, there does seem to be a slight reduction in the extent of axon collapse in double mutants compared with slit alone (Fig. 2D). Together, our phenotypic analysis of compound mutants that simultaneously reduce attraction and repulsion support the idea that these two signaling systems function largely independently in regulating midline crossing.

Neuronal expression of either Robo or Fra in the double-mutant background restores the phenotype to that observed in single mutants

Because our double-mutant data directly contradicts the previously published work of Bhat (2005), we sought additional confirmation of the veracity of our doublemutant strains. Previous studies have established that the axon guidance phenotypes of both robo and fra can be significantly rescued by expressing these genes in postmitotic neurons using the Gal4-UAS system (Kolodziej et al., 1996; Kidd et al., 1998a; Garbe et al., 2006). Therefore, we tested whether transgenic expression of either Fra or Robo in the double mutants would "rescue" the phenotype and restore it to one that more closely resembled the single mutants (Fig. 3). This is clearly the case: reintroducing fra reverts the double mutant to a more robo-like phenotype, whereas reintroducing robo shifts the phenotype back toward fra (Fig. $3 B, C, H, I$ ). In fact, pan-neural

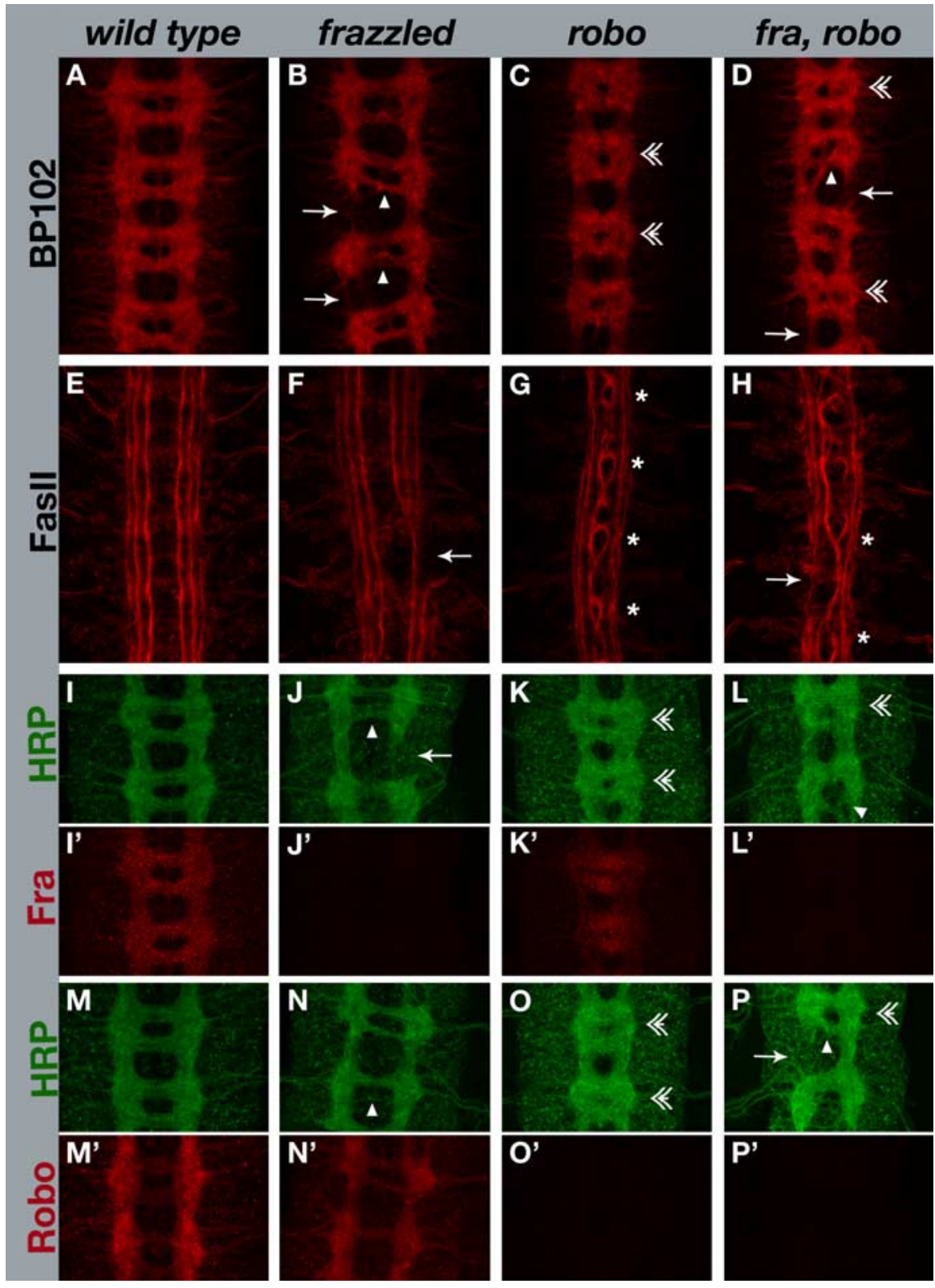

Figure 1. fra, robo double-mutant embryos display a combination of single-mutant phenotypes. For all panels, anterior is up. Arrows represent longitudinal breaks, arrowheads represent thinning of commissures, feathered arrowheads represent condensed/fused commissures, and asterisks represent segments with recrossing longitudinal bundles. Genotypes are listed on top, and antibodies are listed on the left. $\boldsymbol{A}-\boldsymbol{D}$, Stage 16 embryos stained with MAb BP102.A, Wild-type embryos stained with BP102 exhibit a ladder-like CNS scaffold with distinct thick anterior and posterior commissures and continuous longitudinal tracks on each side of the midline. $\boldsymbol{B}$, fra mutant embryos have many segments with thin commissures suggesting reduced midline attraction. fra mutant embryos also display longitudinal breaks. C, robo mutant embryos display fused anterior and posterior commissures. $\boldsymbol{D}$, fra, robo double mutants ( $n>50$ embryos) exhibit a combination of single-mutant phenotypes; for example, thin and/or condensed commissures and longitudinal breaks. $\boldsymbol{E}-\boldsymbol{H}$, Late stage 16-17 embryos stained with anti-Fasll. $\boldsymbol{E}$, In wild-type embryos, three distinct fascicles on each side of the midline never cross and remain ipsilateral. $\boldsymbol{F}$, fra mutants show Iongitudinal breaks of these Fasll-positive fascicles. G, Medial Fasll-positive bundles frequently cross and recross the midline in robo mutants. $\boldsymbol{H}$, fra, robo double mutants ( $\mathrm{n}>50$ embryos) have a combination of single-mutant phenotypes such as longitudinal breaks and medial Fasll-positive bundles crossing the midline. I-L, All embryos stained with anti-HRP (green) to show the axon scaffold and anti-Fra (red). $I_{\boldsymbol{I}} \boldsymbol{I}^{\prime}$, Wild-type embryos have a normal axonal scaffold and are positive for anti-Fra. $\boldsymbol{J}, \boldsymbol{J}^{\prime}$, fra mutant embryos have a disrupted scaffold and are negative for anti-Fra. $\boldsymbol{K}, \boldsymbol{K}^{\prime}$, robo mutants are positive for anti-Fra. $\boldsymbol{L}, \boldsymbol{L}^{\prime}$, fra, robo double mutants are indeed negative for anti-Fra and display a combination of single-mutant phenotypes. $\boldsymbol{M}-\boldsymbol{P}$, All embryos stained with anti-HRP (green) to show the axon scaffold and anti-Robo (red). $\boldsymbol{M}, \boldsymbol{M}^{\prime}$, Wild-type embryos are positive for antiRobo. $\boldsymbol{N}, \boldsymbol{N}^{\prime}$, fra mutants are positive for anti-Robo. $\mathbf{0}, \mathbf{O}^{\prime}$, robo mutants are negative for anti-Robo. $\boldsymbol{P}, \boldsymbol{P}^{\prime}$, fra, robo double-mutant embryos are negative for anti-Robo and show a combination of single-mutant phenotypes. To further confirm the presence of each single mutation in the $\mathrm{fra}^{3}$ or $\mathrm{fra}^{4}, \mathrm{robo} / \mathrm{CyWg}$ stocks, we generated the single-mutant phenotypes in this figure by individually crossing flies from each single mutant to the double-mutant stock. 


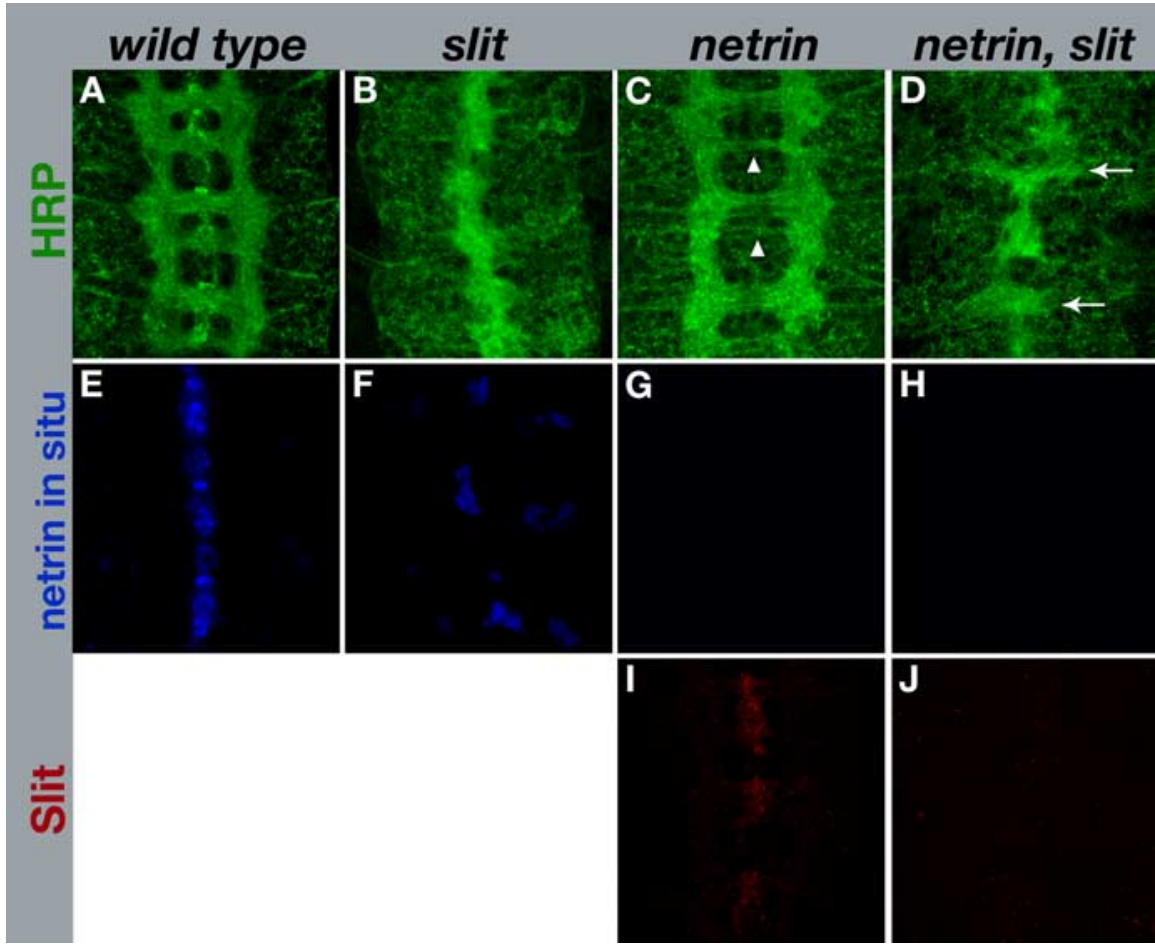

Figure 2. Netrin mutants are not epistatic to slit. $\boldsymbol{A}-\boldsymbol{D}$, Stage 15 animals stained with anti-HRP (green) to reveal all axons. Anterior is up. $\boldsymbol{A}$, Wild-type embryos have a normal axonal scaffold. $\boldsymbol{B}$, Without the repulsive ligand Slit, all axons collapse at the midline. C, Netrin mutant embryos have thinning or absent commissures (arrowheads) consistent with a loss of midline attraction. D, Similar to slit single mutants, in Netrin, slit double mutants ( $n>15$ embryos), all axons collapse at the midline. Some segments appear less severe than slit single mutants, and a broadening of the axon pile at the midline is observed (arrows). $\boldsymbol{E}-\boldsymbol{H}$, Same embryos as in $\boldsymbol{A}-\boldsymbol{D}$ but instead detecting Netrin mRNA (blue). Wild-type $(\boldsymbol{E})$ and slit single mutants $(\boldsymbol{F})$ are positive for Netrin mRNA. In contrast, Netrin single mutants $(\boldsymbol{G})$ and Netrin, slit double mutants $(\boldsymbol{H})$ are negative for Netrin mRNA. Notice that although Netrin, slit double mutants are negative for Netrin mRNA, they still exhibit a slit mutant phenotype. Note also the disorganization of the midline glia in slit mutants in $\boldsymbol{F}$. I, J, Netrin single mutants are positive for Slit, whereas Netrin, slit double mutants are negative, indicating that the double mutants are indeed mutant for slit. Unfortunately, for technical reasons, we could not image the Slit protein in either wild-type or slit single mutants in these triple-labeled embryos because the Cy 5 signal detecting Netrin mRNA interfered with Cy3 (Slit) detection. Notice that the Cy5 "bleed-through" signal is also present in $\boldsymbol{A}$, demonstrating that it was excited by all lasers.

expression of Robo in the fra, robo double mutant not only rescues the ectopic midline crossing but also appears to significantly exacerbate the loss of attraction associated with fra mutants (Fig. 3I).

To further evaluate whether misexpression of Robo can enhance the defects in fra mutants, we misexpressed Robo in all neurons with elavGal4 in either wild-type ( $\mathrm{rra} /+$ heterozygotes) or fra mutants. Consistent with previous data (Kidd et al., 1998b; Bashaw et al., 2000), overexpressing one copy of a UASRoboMyc transgene with elavGal4 in wild-type animals did not lead to significant guidance defects, nor did we observe ectopic Robo protein in the axon commissures (supplemental Fig. 1, available at www.jneurosci.org as supplemental material). In contrast, similar misexpression of UASRoboMyc in fra mutants led to a dramatic disruption in commissure formation, affecting both anterior and posterior commissures (supplemental Fig. 1, available at www.jneurosci.org as supplemental material). The fact that we can enhance the complete loss of fra attraction suggests that panneural expression of Robo repels axons independently of any influence on Netrin-fra signaling, a finding that is consistent with an additive influence of $\mathrm{fra}$ attraction and robo repulsion. Importantly, ectopic Robo expression is unable to repel axons in fra, slit double mutants, arguing that the Robo repulsive gain-of- function phenotype is strictly dependent on slit (supplemental Fig. 1, available at www.jneurosci.org as supplemental material)

In addition to analysis of double mutants, Bhat performed additional genetic experiments to bolster the argument that Slit-Robo signaling functions primarily to inhibit Netrin attraction (Bhat, 2005). In particular, evidence was presented that the gain-of-function ectopic axon attraction phenotype associated with expression of a chimeric receptor consisting of the extracellular domain of Robo and the cytoplasmic domain of Fra (Bashaw and Goodman, 1999) was completely dependent on midline-expressed Netrin. We repeated these experiments with the identical transgenic inserts used in the Bhat study and found no such Netrin dependence: misexpression of the Robo-Fra chimera resulted in ectopic attraction in the presence or absence of Netrin (supplemental Fig. 2, available at www.jneurosci.org as supplemental material).

Together, these observations are inconsistent with the idea that loss of Netrinmediated midline attraction is epistatic to loss of midline repulsion. Our genetic analysis, mRNA and protein expression analysis, and transgenic rescue data leave little doubt that our double mutants remove the relevant gene functions. Importantly, although our data preclude the possibility that slit-robo function is mediated solely through inhibition of Netrin-fra, examination of ipsilateral pioneer posterior corner cell (pCC) neurons in fra, robo or fra, slit double mutants is consistent with the proposal of Hiramoto and Hiromi (2006) that one function of slit-robo signaling is to prevent axons from responding to relocalized Netrin during the pioneering of the longitudinal connectives. In contrast to robo mutants in which the pCC pioneer crosses the midline almost all of the time ( $\sim 96 \%)$, in fra, robo double mutants, this ectopic crossing is significantly reduced to $\sim 60 \%$ (Table 1 ). To extend this observation, we further reduced repulsion in fra mutants by removing slit and found that in $\mathrm{rra}$, slit double mutants there was no significant difference in the extent of pCC ectopic midline crossing compared with slit alone (Table 1). This is likely attributable to the fact that axon commissures do not form at all in slit mutants, and thus Netrin is presumably not relocalized in a way that it can influence the behavior of the longitudinal pioneers (Hiramoto and Hiromi, 2006). Our data are consistent with the idea that Robo signaling likely acts simultaneously to repel pioneers from midline expressed Slit, as well as to prevent longitudinal pioneer axons from responding to Netrin as they navigate the segment boundary.

\section{Robo repels specific subsets of ipsilateral axons} independently of Fra

Although our analysis of simultaneously removing attraction and repulsion clearly reveals aspects of both kinds of phenotypes (es- 
pecially in the case of fra, robo double mutants), the defects observed in late-stage embryos with BP102 and FasII are so severe and affect so many axons that they are very difficult to analyze quantitatively. We were able to circumvent this by quantifying the guidance defects in pCC pioneers in early stages, in which single axon resolution is possible (Table 1 , see above). To attain similar resolution in later navigating ipsilateral neurons, we analyzed the trajectory of small subsets of CNS interneurons in fra and robo single- and double-mutant combinations using apterousGal4 (apGal4) to label three ipsilateral neurons per hemisegment (O'Keefe et al., 1998).

In wild-type or fra mutant embryos, the apterous (ap) neurons in the eight abdominal segments of the embryo project axons toward the midline before turning anteriorly along the outer edge of the medialmost FasII axon bundle; they do not cross the midline (Fig. $4 A, B$ ). In contrast, in robo mutants, the ap neurons extend across the midline and then wander back and forth or stay at the midline, often tracking FasII-positive axon bundles that are ectopically crossing (Fig. 4C). This defect can be rescued by providing robo function specifically in the ap neurons in an otherwise mutant background, indicating that Robo guides later projecting neurons independently of its earlier function in the longitudinal pioneers (Garbe et al., 2006). Interestingly, a time course analysis has shown that midline crossing defects in the ap neurons can arise at two different times. In the complete absence of Robo (or Slit), almost all of the ap axons extend medially, directly across the midline at stage 15, never making their correct anterior turn (Table 1), whereas in embryos mutant for $\beta$-spectrin or carrying genetic combinations that partially limit Slit-Robo signaling, the ap axons initially make the correct ipsilateral anterior turn, but several hours later, at stage 17 , ectopic midline crossing is observed (Garbe et al., 2006). We have interpreted these findings to suggest that Slit-Robo repulsion is required continuously to establish and maintain appropriate pathway selection (Garbe et al., 2006). The early-stage ap axon guidance defect provides an easily quantifiable assay for robo repulsive function. In fra, robo double mutants the majority of ap axons project

directly across the midline, again arguing for fra-independent Robo-mediated midline repulsion (Fig. 4D, Table 1). The observation that the midline crossing defect of the ap neurons in fra, robo double mutants can be rescued by resupplying UASRobo to the ap neurons further supports a fra-independent repulsive function (data not shown). embryos.

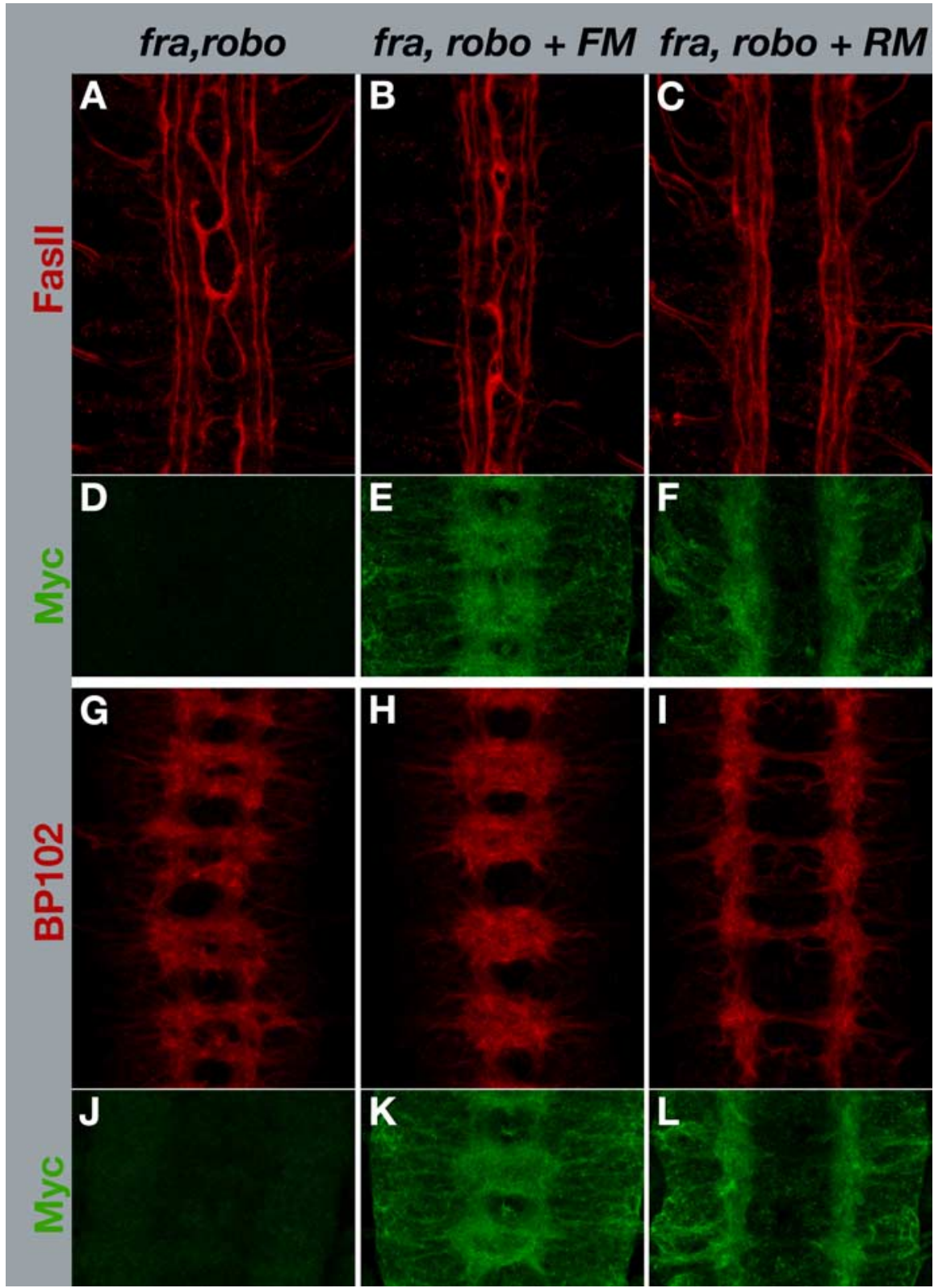

Figure 3. The fra, robo double-mutant phenotypes can be "rescued" by individually expressing each receptor. $\boldsymbol{A}-\boldsymbol{C}$, Late stage 16 embryos stained with anti-Fasll. All embryos are homozygous for fra and robo. Anterior is up. $\boldsymbol{A}$, fra, robo double-mutant embryos exhibit many Fasll-positive axons inappropriately crossing the midline. $\boldsymbol{B}$, More axons seem to ectopically cross and stay at the midline when Fra is misexpressed in all axons ( $n=16$ embryos). $C$, When Robo is overexpressed in all axons, Fasll-positive neurons are rescued and no longer cross the midline $(n=12$ embryos). $\boldsymbol{D}-\boldsymbol{F}$, Anti-Myc staining demonstrating UASFra-Myc (FM) $(\boldsymbol{E})$ or UASRobo-Myc $(R M)(\boldsymbol{F})$ in the above embryos. $\mathbf{G}-\mathbf{I}$, Mid-stage 16 embryos stained with MAb BP102. All embryos are homozygous for fra and robo. Anterior is up. G, fra, robo double mutants show a combination of single-mutant phenotypes; for example, condensed and/or thinning commissures and longitudinal breaks. $\boldsymbol{H}$, The double-mutant phenotype can be rescued back to robo single mutants by expressing Fra in all neurons $(n=17$ embryos). Often, this phenotype seems qualitatively stronger than robo single mutants suggesting Fra can attract additional axons independently of Robo. I, When Robo is expressed in the fra, robo double-mutant background, the phenotype is rescued back to the fra single-mutant phenotype ( $n=18$ embryos). Often, this phenotype is considerably more severe than fra single mutants, suggesting that Robo is able to repel commissural axons independently of fra. $\boldsymbol{J}-\boldsymbol{L}$, Anti-Myc staining demonstrating UASFra-Myc (FM) (K) or UASRobo-Myc $(R M)(\boldsymbol{L})$ in the above

\section{Commissural axons recross the midline in robo mutants in the absence of fra}

To more clearly define the role of Netrin-fra attraction and slitrobo repulsion in the guidance of precrossing and postcrossing commissural axons, we next examined the trajectories of the Egl commissural interneurons in different single- and double- 
Table 1. Quantification of midline crossing defects

\begin{tabular}{|c|c|c|c|c|}
\hline \multirow[b]{2}{*}{ Genotype } & \multicolumn{2}{|l|}{ Ipsilateral axon projections } & \multicolumn{2}{|c|}{ Contralateral axon projections } \\
\hline & 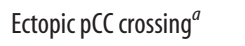 & Ectopic ap crossing $^{b}$ & EW crossing defects $^{c}$ & EG recrossing $^{d}$ \\
\hline Wild type & $0 / 88(0 \%) n=8$ & $0 / 99(0 \%) n=9$ & $0 / 96(0 \%) n=12$ & $0 / 96(0 \%) n=12$ \\
\hline fra & $0 / 110(0 \%) n=10$ & $0 / 88(0 \%) n=8$ & $257 / 372(69 \%) n=49$ & $0 / 80(0 \%) n=10$ \\
\hline robo & $143 / 147(97 \%) n=15$ & $206 / 217(95 \%) n=23$ & $0 / 96(0 \%) n=12$ & $134 / 208(64 \%) n=26$ \\
\hline fra, robo & $\begin{array}{l}115 / 186(61 \%)^{*} n=19 \\
p=6.15 \mathrm{e}-07\end{array}$ & $\begin{array}{l}162 / 193(84 \%)^{*} n=19 \\
p=0.0001\end{array}$ & $\begin{array}{l}163 / 307(53 \%)^{* *} n=41 \\
p=8.60 \mathrm{e}-05\end{array}$ & $\begin{array}{l}107 / 232(46 \%)^{*} n=18 \\
p=9.02 \mathrm{e}-06\end{array}$ \\
\hline slit & $166 / 176(94 \%) n=18$ & ND & ND & ND \\
\hline fra, slit & $313 / 351(89 \%) n=36$ & ND & ND & ND \\
\hline
\end{tabular}

In all cases, defects are indicated as number of defective segments divided by the total segments scored to give a percentage. $n$ represents the number of embryos of the specified genotype that were analyzed. ${ }^{~}$ Statistically different from robo alone or **fra alone in a two-sample Student's $t$ test with $p<0.01$. $p$ values are indicated. ND, Not determined.

${ }^{a}$ Late stage 12 to early stage 13 embryos stained with anti-Fas 2 were scored.

${ }^{b}$ Stage 15 embryos in which the ap neurons were labeled with GFP were scored for ectopic crossing defects.

'Stage 16 embryos in which the EW neurons were labeled with GFP were scored for defects in normal midline crossing. Defects include a complete loss of or a noticeable thinning of the commissural EW bundle. Only the eight abdominal segments were scored.

${ }^{d}$ Stage 17 embryos in which the EG neurons were labeled with GFP were scored for abnormal recrossing at the midline.

mutant combinations. In wild-type embryos, the Egl neurons comprise two clusters of neurons: one cluster (the EG neurons) contains $10-12$ cells that extend their axons across the midline in the anterior commissure, and the second cluster (the EW neurons) consists of four cells, three of which are serotonergic interneurons that project axons across the midline in the posterior commissure (the other cell is a motor neuron) (Fig. 4E) (Higashijima et al., 1996; Dittrich et al., 1997). Lineage tracing has established that the EW neurons are derived from neuroblast 7-3 and that they make local synaptic connections immediately after crossing the midline, whereas the EG commissural interneurons are derived from neuroblast 3-3 and that they cross the midline and then project anteriorly for several segments traveling near the medial edge of the longitudinal connective (Fig. 4E,I) (Bossing et al., 1996; Schmidt et al., 1997; Schmid et al., 1999). In fra mutants, the EW neurons frequently fail to cross the midline, whereas the EG axonal projections in the anterior commissure are unaffected both precrossing and postcrossing (Fig. $4 F, J$, Table 1). In contrast, robo mutants exhibit defects that are most prominent in postcrossing EG neurons, in which inappropriate recrossing and circling around the midline are observed in late-stage embryos (Fig. 4G,K). Interestingly, in fra, robo double mutants, there is a small but significant decrease in the number of EW axons that fail to cross the midline compared with fra single mutants, suggesting that in a wild-type situation this repulsive function of Robo in precrossing commissural EW axons may be counterbalanced by fra attraction (Fig. 4H, Table 1). Similarly, postcrossing EG axons exhibit a slight reduction in midline recrossing and circling behavior compared with robo single mutants (Fig. $4 L$, Table 1). We believe that these subtle shifts in the percentage of defects in the double mutants reflect alterations in the balance of attraction and repulsion.

\section{Discussion}

Here, we have investigated how attraction and repulsion are coordinated during midline axon guidance in Drosophila to determine whether Slit and Robo function to mediate repulsion or alternatively whether they function to inhibit Netrin-Fra attraction. We generated double mutants that simultaneously remove the attractive and repulsive ligands and receptors and verified the absence of each of the proteins in our double mutants using molecular and genetic criteria. Analysis of their phenotypes using markers for large groups of axons, as well as markers for specific subsets of ipsilateral and contralateral neurons, reveals a combination of guidance defects that include aspects of both singlemutant phenotypes. Furthermore, gain-of-function experiments indicate that Robo can repel CNS axons in the absence of fra function, arguing for an independent repulsive function of Slit and Robo. In addition, we found that postcrossing commissural neurons recross the midline in robo mutants, and this behavior is also largely independent of Netrin-fra attraction. Together, our data support at least two distinct functions for Slit-Robo signaling in the regulation of midline crossing; one role to repress Netrin-fra function during the guidance of longitudinal pioneer neurons at the segment boundary and a second major Netrin-fra-independent repulsive role in preventing ectopic midline crossing.

\section{Independent functions of Netrin-Fra and Slit-Robo regulate midline crossing}

Our study was inspired in part by the provocative findings of Bhat (2005) indicating that mutations in Netrin and fra were epistatic to mutations in slit and robo; that is, compound mutants between components of these two signaling pathways resulted in phenotypes indistinguishable from single mutants in fra or Netrin. These observations were surprising because loss of slit function leads to profound guidance defects in essentially all CNS axons, whereas loss of Netrin or fra causes much milder defects (Harris et al., 1996; Kolodziej et al., 1996; Mitchell et al., 1996; Bashaw and Goodman, 1999; Hummel et al., 1999; Forsthoefel et al., 2005; Brankatschk and Dickson, 2006). Indeed, many commissural neurons cross the midline normally in the absence of $\mathrm{Ne}$ trin-fra signaling. So how could a mutation that affects only some neurons be epistatic to a mutation that affects all neurons? The data presented here directly contradict the published doublemutant analysis (Bhat, 2005) and support the more parsimonious explanation that Robo repels axons independently of NetrinFra attraction.

Here, it should be noted that several clear examples of independent repulsive functions for Slit and Robo have been described in both C. elegans and Vertebrates (Brose and TessierLavigne, 2000; Zou et al., 2000; Stein and Tessier-Lavigne, 2001; Yu et al., 2002). Furthermore, studies in C. elegans have suggested that Slit repulsion and Netrin attraction act cooperatively during ventral guidance and that DCC/UNC-40 can actually potentiate SAX-3/Robo repulsion independently of Unc-6/Netrin (Hao et al., 2001; Yu et al., 2002). The most likely explanation for the discrepancy between our data and that reported by Bhat is the misidentification of the double-mutant embryos in the earlier study.

\section{The role of Robo in longitudinal pioneer axons}

Hiramoto and Hiromi have observed that the abnormal midline crossing of specific pioneer ipsilateral neurons seen in robo mu- 
tants can be strongly suppressed by simultaneous removal of Netrin or fra, suggesting that the robo defect results from a failure to inhibit a response to redistributed Netrin, rather than a loss of midline repulsion (Hiramoto et al., 2000; Hiramoto and Hiromi, 2006). In this context, Robo is proposed to function to prevent longitudinally extending pioneer axons from responding to Netrin that has been relocalized to commissural axons through interactions with Fra (Hiramoto et al., 2000). We also observed a significant reduction in pioneer crossing in fra, robo double mutants compared with robo single mutants, suggesting that defects in longitudinal pioneers are not strictly caused by loss of repulsion. To investigate this possibility further, we also determined the incidence of longitudinal pioneer defects in fra, slit double mutants, a background in which all Slit-dependent repulsion is eliminated. In contrast to fra, robo double mutants, fra, slit double mutants exhibited the same high levels of ectopic crossing as observed in slit single mutants, indicating that completely removing midline repulsion masks the function of Robo in preventing abnormal responses to redistributed Netrin. Together, our genetic results suggest that Slit-Robo signaling likely acts simultaneously to repel pioneer neurons from midline-expressed Slit, as well as to prevent longitudinal pioneer axons from responding to Netrin as they navigate the segment boundary.

\section{Guidance at the Drosophila midline:} balancing attraction and repulsion or hierarchical receptor interactions? It has been proposed that navigating growth cones measure the relative levels of attractive and repulsive cues to arrive at the correct decisions (Tessier-Lavigne and Goodman, 1996). In this scenario, the relative influence of attractive and repulsive cues can be biased in one direction or another by regulating the complement of guidance receptors that are expressed on the surface of the growth cone. Experimental manipulations in which the levels of guidance cues and receptors are either increased or decreased, singly or in combinations, have provided substantial evidence in support of this idea; in particular in the context of selective axon fasciculation, midline axon guidance, and target selection (Lin et al., 1994; Tessier-Lavigne and Goodman, 1996; Winberg et al., 1998; Bashaw and Goodman, 1999). Recent work has suggested an alternative (although certainly not mutually exclusive) idea that at certain times during the trajectory of an axon, attractive and repulsive signals may be more intimately connected, with one response overriding another through direct receptor-receptor interactions (Stein and Tessier-Lavigne, 2001).

A number of findings presented here support the model that the relative levels of attractive and repulsive influences play an important role in instructing the decision of whether or not to cross the midline. First, our observation that simultaneous removal of attraction and repulsion leads to defects that combine aspects of removing either one supports the idea that these two signaling systems act independently. Furthermore, the fact that pan-neural misexpression of robo in the absence of fra (or in fra, robo double mutants) results in much stronger disruptions in midline crossing than when fra is present argues that (1) Robo can repel additional axons independently of Fra attraction, and (2) that the ability of Robo to ectopically repel axons can be counteracted by the independent attractive influence of Fra. Similarly, misexpression of fra in the fra, robo double mutant results in midline crossing defects that appear to be qualitatively more severe than robo single mutants, whereas misexpression of fra in wild-type embryos has no effect (data not shown), again suggesting that ectopic attraction mediated by fra can be counteracted by the independent repulsive function of Robo. 


\section{Implications for silencing}

The "silencing" model provides an elegant explanation for how a postcrossing commissural neuron might couple the upregulation of Robo repulsion to the downregulation of attraction to ensure high-fidelity guidance (Stein and Tessier-Lavigne, 2001) and is consistent with the finding that postcrossing commissural hindbrain neurons lose responsiveness to Netrin (Shirasaki et al., 1998). Our observation that postcrossing commissural axons in robo mutants can recross the midline in the absence of framediated axon attraction has important implications for the mechanism by which commissural axons normally avoid recrossing. Under its strictest interpretation, the silencing model proposed by Stein and Tessier-Lavigne posits that postcrossing commissural neurons require Slit-Robo function to downregulate the attractive response to midline-expressed Netrin and that this function is essential to prevent recrossing (Stein and TessierLavigne, 2001). The fact that the majority of EG commissural axons recross in the absence of fra function would suggest that at the Drosophila midline, silencing of fra is not absolutely required to prevent recrossing and therefore would not be consistent with the extreme interpretation of silencing as stated above. Here it is important to point out that despite considerable conservation in the molecules and mechanisms mediating midline guidance in vertebrates and invertebrates, there are significant differences in the two systems. In particular, the mechanism of Robo regulation in precrossing axons appears to be distinct in the two systems, with the Commissureless protein inhibiting Robo function in commissural axons in the fly, whereas the variant Robo family member Rig-1 appears to fulfill this role in vertebrates (Kidd et al., 1998b; Keleman et al., 2002; Sabatier et al., 2004).

Therefore, we propose two mutually exclusive possibilities: (1) either Robo silencing of Fra does not occur at the Drosophila midline (or at least not in these particular commissural neurons), or (2) silencing does occur, but either Robo repulsion on its own is sufficient to prevent recrossing, or there are additional attractive functions that must also be silenced in postcrossing axons. Currently, we are unable to distinguish between these possibilities, although our previous observation that Robo repulsion is required continuously throughout embryonic development to maintain appropriate pathway selection, together with the double-mutant analyses we describe here, support an independent repulsive function of Robo in regulating axon crossing at the midline. Future studies in Drosophila and Vertebrates, for example, with the analysis of mice bearing mutations in both Robo and DCC receptors, should shed additional light on how much of a role silencing plays during midline guidance in vivo.

\section{References}

Bashaw GJ, Goodman CS (1999) Chimeric axon guidance receptors: the cytoplasmic domains of slit and netrin receptors specify attraction versus repulsion. Cell 97:917-926.

Bashaw GJ, Kidd T, Murray D, Pawson T, Goodman CS (2000) Repulsive axon guidance: Abelson and Enabled play opposing roles downstream of the roundabout receptor. Cell 101:703-715.

Bhat KM (2005) Slit-roundabout signaling neutralizes netrin-Frazzledmediated attractant cue to specify the lateral positioning of longitudinal axon pathways. Genetics 170:149-159.

Bossing T, Udolph G, Doe CQ, Technau GM (1996) The embryonic central nervous system lineages of Drosophila melanogaster. I. Neuroblast lineages derived from the ventral half of the neuroectoderm. Dev Biol 179:41-64.

Brankatschk M, Dickson BJ (2006) Netrins guide Drosophila commissural axons at short range. Nat Neurosci 9:188-194.

Brose K, Tessier-Lavigne M (2000) Slit proteins: key regulators of axon guidance, axonal branching, and cell migration. Curr Opin Neurobiol 10:95-102.
Brose K, Bland KS, Wang KH, Arnott D, Henzel W, Goodman CS, TessierLavigne M, Kidd T (1999) Slit proteins bind Robo receptors and have an evolutionarily conserved role in repulsive axon guidance. Cell 96:795-806.

Dickson BJ (2001) Rho GTPases in growth cone guidance. Curr Opin Neurobiol 11:103-110.

Dittrich R, Bossing T, Gould AP, Technau GM, Urban J (1997) The differentiation of the serotonergic neurons in the Drosophila ventral nerve cord depends on the combined function of the zinc finger proteins Eagle and Huckebein. Development 124:2515-2525.

Forsthoefel DJ, Liebl EC, Kolodziej PA, Seeger MA (2005) The Abelson tyrosine kinase, the Trio GEF and Enabled interact with the Netrin receptor Frazzled in Drosophila. Development 132:1983-1994.

Garbe D, Bashaw G (2004) Axon guidance at the midline: from mutants to mechanisms. Crit Rev Biochem Mol Biol 39:319-341.

Garbe DS, Das A, Dubreuil RR, Bashaw GJ (2006) beta-Spectrin functions independently of Ankyrin to regulate the establishment and maintenance of axon connections in the Drosophila embryonic CNS. Development 134:273-284.

Hao JC, Yu TW, Fujisawa K, Culotti JG, Gengyo-Ando K, Mitani S, Moulder G, Barstead R, Tessier-Lavigne M, Bargmann CI (2001) C. elegans slit acts in midline, dorsal-ventral, and anterior-posterior guidance via the SAX-3/Robo receptor. Neuron 32:25-38.

Harris R, Sabatelli LM, Seeger MA (1996) Guidance cues at the Drosophila CNS midline: identification and characterization of two Drosophila Netrin/UNC-6 homologs. Neuron 17:217-228.

Higashijima S, Shishido E, Matsuzaki M, Saigo K (1996) eagle, a member of the steroid receptor gene superfamily, is expressed in a subset of neuroblasts and regulates the fate of their putative progeny in the Drosophila CNS. Development 122:527-536.

Hiramoto M, Hiromi Y (2006) ROBO directs axon crossing of segmental boundaries by suppressing responsiveness to relocalized Netrin. Nat Neurosci 9:58-66.

Hiramoto M, Hiromi Y, Giniger E, Hotta Y (2000) The Drosophila Netrin receptor Frazzled guides axons by controlling Netrin distribution. Nature 406:886-889.

Hummel T, Schimmelpfeng K, Klambt C (1999) Commissure formation in the embryonic CNS of Drosophila. Development 126:771-779.

Keleman K, Rajagopalan S, Cleppien D, Teis D, Paiha K, Huber LA, Technau GM, Dickson BJ (2002) Comm sorts robo to control axon guidance at the Drosophila midline. Cell 110:415-427.

Kidd T, Brose K, Mitchell KJ, Fetter RD, Tessier-Lavigne M, Goodman CS, Tear G (1998a) Roundabout controls axon crossing of the CNS midline and defines a novel subfamily of evolutionarily conserved guidance receptors. Cell 92:205-215.

Kidd T, Russell C, Goodman CS, Tear G (1998b) Dosage sensitive and complementary functions of Roundabout and Commissureless control axon crossing of the CNS midline. Neuron 20:25-33.

Kidd T, Bland KS, Goodman CS (1999) Slit is the midline repellent for the robo receptor in Drosophila. Cell 96:785-794.

Kolodziej PA, Timpe LC, Mitchell KJ, Fried SR, Goodman CS, Jan LY, Jan YN (1996) frazzled encodes a Drosophila member of the DCC immunoglobulin subfamily and is required for CNS and motor axon guidance. Cell 87:197-204.

Lin DM, Fetter RD, Kopczynski C, Grenningloh G, Goodman CS (1994) Genetic analysis of fasciclin II in Drosophila: defasciculation, refasciculation, and altered fasciculation. Neuron 13:1055-1069.

Long H, Sabatier C, Ma L, Plump A, Yuan W, Ornitz DM, Tamada A, Murakami F, Goodman CS, Tessier-Lavigne M (2004) Conserved roles for Slit and Robo proteins in midline commissural axon guidance. Neuron 42:213-223.

Mitchell KJ, Doyle JL, Serafini T, Kennedy TE, Tessier-Lavigne M, Goodman CS, Dickson BJ (1996) Genetic analysis of Netrin genes in Drosophila: Netrins guide CNS commissural axons and peripheral motor axons. Neuron 17:203-215.

O'Keefe DD, Thor S, Thomas JB (1998) Function and specificity of LIM domains in Drosophila nervous system and wing development. Development 125:3915-3923.

Rajagopalan S, Nicolas E, Vivancos V, Berger J, Dickson BJ (2000) Crossing the midline: roles and regulation of Robo receptors. Neuron 28:767-777.

Sabatier C, Plump AS, Le M, Brose K, Tamada A, Murakami F, Lee EY, Tessier-Lavigne M (2004) The divergent Robo family protein rig-1/ 
Robo3 is a negative regulator of slit responsiveness required for midline crossing by commissural axons. Cell 117:157-169.

Schmid A, Chiba A, Doe CQ (1999) Clonal analysis of Drosophila embryonic neuroblasts: neural cell types, axon projections and muscle targets. Development 126:4653-4689.

Schmidt H, Rickert C, Bossing T, Vef O, Urban J, Technau GM (1997) The embryonic central nervous system lineages of Drosophila melanogaster. II. Neuroblast lineages derived from the dorsal part of the neuroectoderm. Dev Biol 189:186-204.

Serafini T, Kennedy TE, Galko MJ, Mirzayan C, Jessell TM, Tessier-Lavigne M (1994) The netrins define a family of axon outgrowth-promoting proteins homologous to C. elegans UNC-6. Cell 78:409-424.

Serafini T, Colamarino SA, Leonardo ED, Wang H, Beddington R, Skarnes WC, Tessier-Lavigne M (1996) Netrin-1 is required for commissural axon guidance in the developing vertebrate nervous system. Cell 87:1001-1014.

Shirasaki R, Katsumata R, Murakami F (1998) Change in chemoattractant responsiveness of developing axons at an intermediate target. Science 279:105-107.

Simpson JH, Kidd T, Bland KS, Goodman CS (2000) Short-range and longrange guidance by slit and its Robo receptors. Robo and Robo2 play distinct roles in midline guidance. Neuron 28:753-766.
Stein E, Tessier-Lavigne M (2001) Hierarchical organization of guidance receptors: silencing of netrin attraction by slit through a Robo/DCC receptor complex. Science 291:1928-1938.

Tear G, Harris R, Sutaria S, Kilomanski K, Goodman CS, Seeger MA (1996) commissureless controls growth cone guidance across the CNS midline in Drosophila and encodes a novel membrane protein. Neuron 16:501-514.

Tessier-Lavigne M, Goodman CS (1996) The molecular biology of axon guidance. Science 274:1123-1133.

Winberg ML, Mitchell KJ, Goodman CS (1998) Genetic analysis of the mechanisms controlling target selection: complementary and combinatorial functions of netrins, semaphorins, and IgCAMs. Cell 93:581-591.

Yu TW, Bargmann CI (2001) Dynamic regulation of axon guidance. Nat Neurosci 4 [Suppl]:1169-1176.

Yu TW, Hao JC, Lim W, Tessier-Lavigne M, Bargmann CI (2002) Shared receptors in axon guidance: SAX-3/Robo signals via UNC-34/Enabled and a Netrin-independent UNC-40/DCC function. Nat Neurosci 5:1147-1154.

Zou Y, Stoeckli E, Chen H, Tessier-Lavigne M (2000) Squeezing axons out of the gray matter: a role for slit and semaphorin proteins from midline and ventral spinal cord. Cell 102:363-375. 